Spin Physics (SPIN2014)

International Journal of Modern Physics: Conference Series

Vol. 40 (2016) 1660004 (13 pages)

(C) The Author(s)

DOI: 10.1142/S2010194516600041

\title{
Physics with Polarized Targets in Storage Ring
}

\author{
Toporkov Dmitriy \\ Budker Institute of Nuclear Physics, \\ Novosibirsk State University, \\ Novosibirsk 630090, Russia \\ D.K.Toporkov@inp.nsk.su
}

Published 29 February 2016

\begin{abstract}
The method of experiments with internal targets in the storage ring was proposed at the moment of creation of the first storage ring of charged particles. This method has demonstrated dramatic advances during the last 50 years. The most clearly visible progress can be seen in experiments with polarized gas targets. In this report we shall review: (a) the experiments on nuclear physics in the storage rings on the measure of polarization observables in different reactions with electron/positron and proton/deuteron beams, (b) the use of polarized gas targets in the storage ring for polarimetry of the circulated beams. The present status of the activity in this field of experimental physics is given.
\end{abstract}

Keywords: Storage ring of charged particles; polarized target.

PACS numbers: 13.40.Gp, 29.27.Hj, 29.25.Pj, 29.20.db

\section{Introduction}

The method of experiments with internal targets in the storage ring of charged particles was proposed at the moment of creation of the first storage rings. This method was first realized in series of experiments which were conducted at the VEPP-2 electron storage ring to study the properties of light nuclei with coincidence detection of the scattered electron and nuclear decay products, including the slow particles in the late 1960s at the Institute of Nuclear Physics, Novosibirsk. ${ }^{1,2}$ Soon afterward this method was widely employed in many accelerator laboratories over the world. Almost all advantages of the internal target technique were realized during the succeeding period. The most clearly visible success can be seen in experiments with internal polarized gas targets. The marvelous results have been achieved in the experiments on the measurement of all deuteron electromagnetic form factors, electric form factor of the neutron, on the measurements of the tensor analyzing powers

This is an Open Access article published by World Scientific Publishing Company. It is distributed under the terms of the Creative Commons Attribution 3.0 (CC-BY) License. Further distribution of this work is permitted, provided the original work is properly cited. 


\section{T. Dmitriy}

in deuteron photodisintegration. The HERMES experiment at HERA, DESY successfully explored the spin structure of the nucleon. A polarized proton target used as a spin filter at TSR demonstrated a possibility to obtain a polarized circulated proton (in future antiproton) beam. Large number of experiments were performed in ion storage rings with polarized targets and polarized electron-cooled proton or deuteron beams. The goal of these experiments was to measure the analyzing power and spin correlation coefficients in the reactions under the study. A polarized hydrogen jet target was used for the measurement of polarization of circulated protons beam at RHIC. Also a polarized hydrogen jet target having polarized electrons was used at VEPP-3 storage ring to measure the polarization of the circulated electron beam. A list of publications with the use of polarized gas targets in storage rings is so big that it is impossible to mention all of them. I have to omit something, I apologize for that.

\section{Experiments with Polarized Targets in the Electron Storage Rings}

\subsection{Electromagnetic structure of the deuteron}

The deuteron is the simplest nucleus and the description of its internal structure is a standard test for nuclear theory. In one photon exchange approximation the electromagnetic structure of the deuteron is completely described by the three form factors: the charge monopole, $G_{c}\left(Q^{2}\right)$, charge quadrupole, $G_{Q}\left(Q^{2}\right)$, and magnetic dipole, $G_{M}\left(Q^{2}\right)$, ones, which depend only on the momentum transfer $Q$ squared. The two deuteron structure functions, $A=G_{C}^{2}+8 / 9 \tau^{2} G_{Q}^{2}+2 / 3 \tau G_{M}^{2}$ and $B=$ $4 / 3 \tau(1+\tau) G_{M}^{2}$, where $\tau=Q^{2} / 4 M_{d}^{2}$ and $M_{d}$ is the deuteron mass, can be found from a series of measurements of the differential cross sections $d \sigma / d \Omega$

$$
\frac{d \sigma}{d \Omega}=\frac{d \sigma_{0}}{d \Omega}\left\{A+B \tan ^{2}\left(\theta_{e} / 2\right)\right\}
$$

for elastic unpolarized ed scattering under various kinematic conditions and the same $Q^{2}, \theta_{e}$ - is a scattering angle. ${ }^{3,4}$ To separate the charge monopole and quadrupole form factors requires at least one more independent measurement. This additional measurement measurement can be achieved with a tensor-polarized deuterium target.

In such a case the polarization observables (analyzing powers) of the reaction of the elastic scattering are dependent on the form factors of the deuteron and all of them can be extracted separately. The first use of internal tensor polarized deuteron target was realized in 1985 in an electron storage ring VEPP-2. ${ }^{5}$ Later similar measurements with internal tensor polarized target in the range of higher momentum transfer were carried out at VEPP-3. ${ }^{6,7}$ NIKHEF AmPSM ${ }^{8,9}$ and the MIT Bates Linear Accelerator Center. ${ }^{10}$ The results on the separated $G_{c}$ and $G_{Q}$ are shown in Fig. 1 including the data obtained with fix solid target. The fit of the all data including ${ }^{7,11}$ confirms the location of the first node of $G_{c}$ at $4.19 \pm 0.05 \mathrm{fm}^{-1}$, consistent with previous results. 

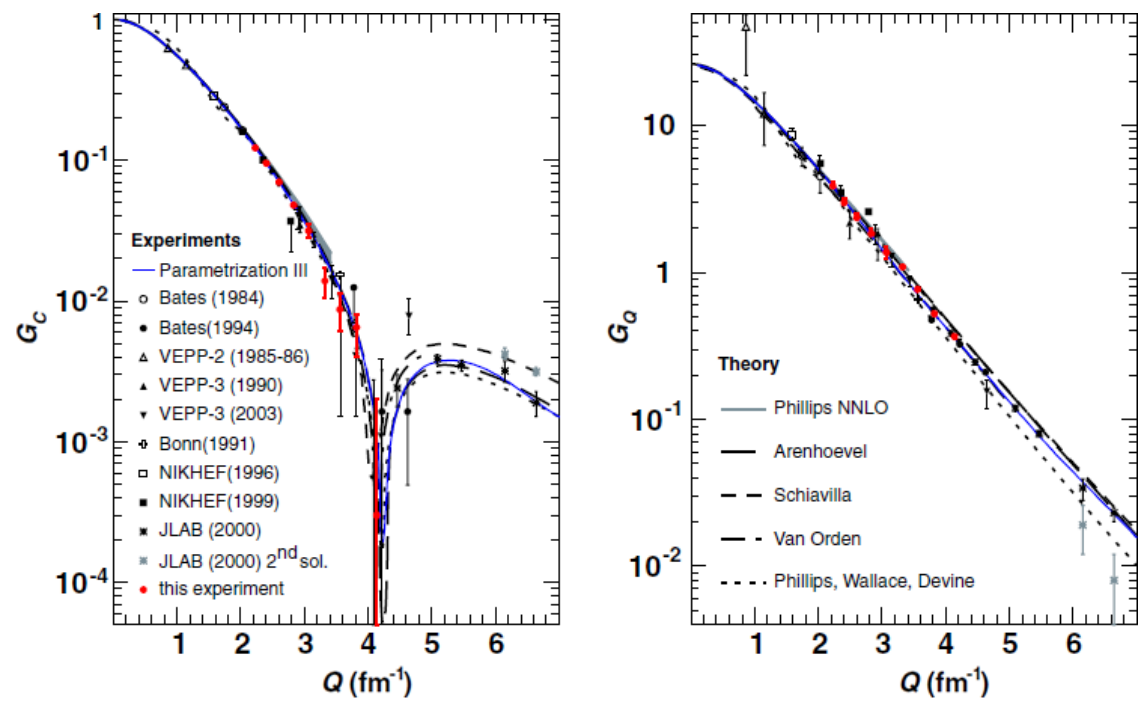

Fig. 1. Results on separated $G_{C}$ and $G_{Q}$ compared to various theoretical predictions. From Ref. 10 .

\subsection{The Charge form factor of the neutron}

Although the neutron has no net electric charge, it does have a charge distribution. The measurements with thermal neutrons have shown that the neutron has a positive core surrounded by a region of negative charge. The actual distribution is described by the charge form factor $G_{n}^{E}$, which enters the cross section for the elastic electron scattering. Due to the lack of the pure neutron target the information on neutron form factors can be found in experiments with ${ }^{2} \mathrm{H}$ or ${ }^{3} \mathrm{He}$ targets. This is especially true if the targets are polarized. The differential cross section for the ${ }^{2} \vec{H}\left(\vec{e}, e^{\prime} n\right) p$ reaction with polarized beam and target can be written

$$
\frac{d^{3} \sigma}{d \Omega_{e} d \Omega_{p q} d \omega}=\sigma_{u n p}\left(1+\Sigma+P_{e} \Delta\right)
$$

with $\Sigma=\sqrt{ } 3 / 2 P_{z} A_{d}^{V}+\sqrt{ } 1 / 2 P_{z z} A_{d}^{T}, \Delta=A_{e}+\sqrt{ } 3 / 2 P_{z} A_{\text {ed }}^{V}+\sqrt{ } 1 / 2 P_{z z} A_{\text {ed }}^{T}$, where $\sigma_{u n p}$ is the unpolarized differential cross section, $P z$ and $P z z$ are the vector and tensor polarizations of the deuteron target, $P_{e}$ is the longitudinal polarization of the electron beam and $A_{i}^{j}$ are spin-correlation observables. The beam-target vector polarization observable $A_{e d}^{V}$ is particularly sensitive to $G_{n}^{E}$ in the quasi-elastic neutron-knockout kinematics. For the first time the neutron electric form factor in the reaction ${ }^{2} \vec{H}\left(\vec{e}, e^{\prime} n\right) p$ were measured at NIKHEF (Amsterdam). ${ }^{12}$ Later new measurements of the neutron charge form factor in a wider range of momentum transfer using quasielastic electrodisintegration of the deuteron were performed at the MIT-Bates Linear Accelerator Center. ${ }^{13}$ The BLAST detector was used to detect quasielastically scattered electrons in coincidence with recoil neutrons over a range of $Q^{2}$ between 0.10 and $0.55(\mathrm{GeV} / \mathrm{c}){ }^{2}$ 


\section{T. Dmitriy}
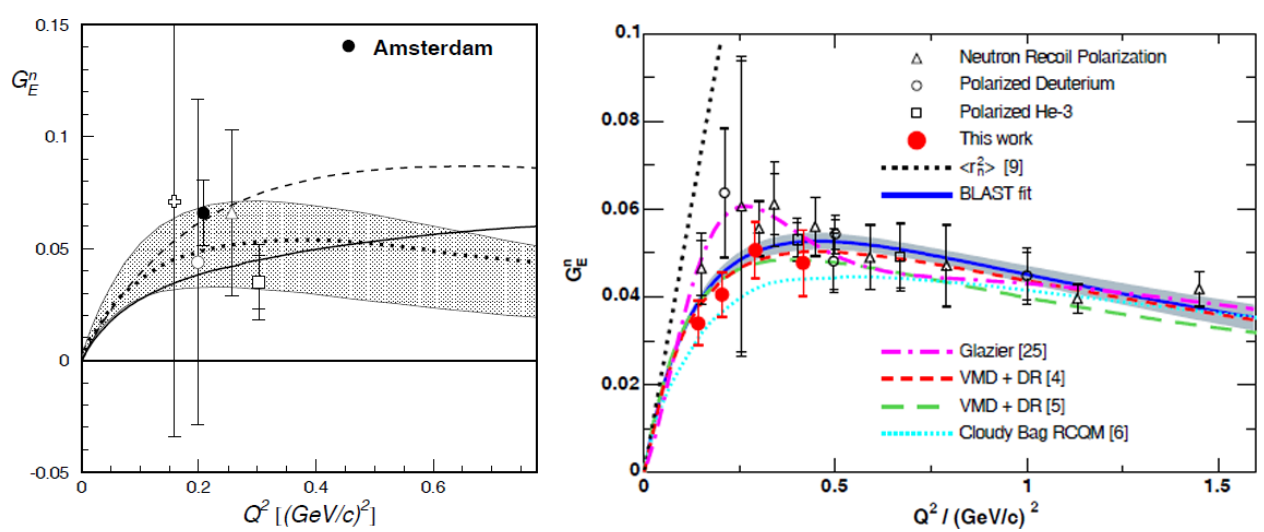

Fig. 2. World data on $G_{n}^{E}$ from double-polarization experiments. Left from Ref. 12, right from Ref. 13.

Also the first precision measurement of the proton electric to magnetic form factor ratio from spin-dependent elastic scattering of longitudinally polarized electrons from a polarized hydrogen internal gas target were performed at MIT-Bates Linear Accelerator Center. ${ }^{14}$ The measurement covered the range of four-momentum transfer squared $Q^{2}$ from 0.15 to $0.65(\mathrm{GeV} / \mathrm{c}){ }^{2}$

\subsection{Photodisintegration of polarized deuteron}

One of the most fundamental processes on the deuteron is two-body photodisintegration $(\mathrm{PD}) \gamma+d \rightarrow p+n$. It has been a subject of intensive experimental and theoretical research for many years. ${ }^{15}$ However several important observables still are measured with insufficient accuracy or not measured at all. The tensor analyzing powers of the reaction accessible through measurement of target asymmetries are among the most poorly known. They are especially interesting because there is a correlation between the degree of tensor polarization and the spatial alignment of the deuteron. Polarization observables are expected to be sensitive to important dynamical details and thus allow in general much more stringent tests of theoretical models. Also the induced recoil proton polarization is consistent with zero above $1 \mathrm{GeV}$ photon energy in disagreement with meson-baryon calculations, see Ref. 15 . It's very interesting to investigate if similar effect exists in tensor analyzing powers. A general expression for the cross-section of the two-body PD of the polarized deuteron is written as follows:

$$
\begin{aligned}
\frac{d \sigma}{d \Omega}= & \frac{d \sigma_{0}}{d \Omega}\left\{1-\sqrt{ } 3 / 4 P_{z} \sin \theta_{H} \sin \varphi_{H} T_{11}+\sqrt{ } 1 / 2 P_{z z}\left\{\left(3 / 2 \cos ^{2} \theta_{H}-1 / 2\right) T_{20}\right.\right. \\
& \left.\left.+\sqrt{ } 3 / 8 \sin 2 \theta_{H} \cos \varphi_{H} T_{21}+\sqrt{ } 3 / 8 \sin ^{2} \theta_{H} \cos 2 \varphi_{H} T_{22}\right\}\right\}
\end{aligned}
$$

with $\sigma_{0}$ the unpolarized cross-section, $P_{z}\left(P_{z z}\right)$ the degree of vector (tensor) polarization of the target, $\theta_{H}$ the angle between polarization axis and momentum of 

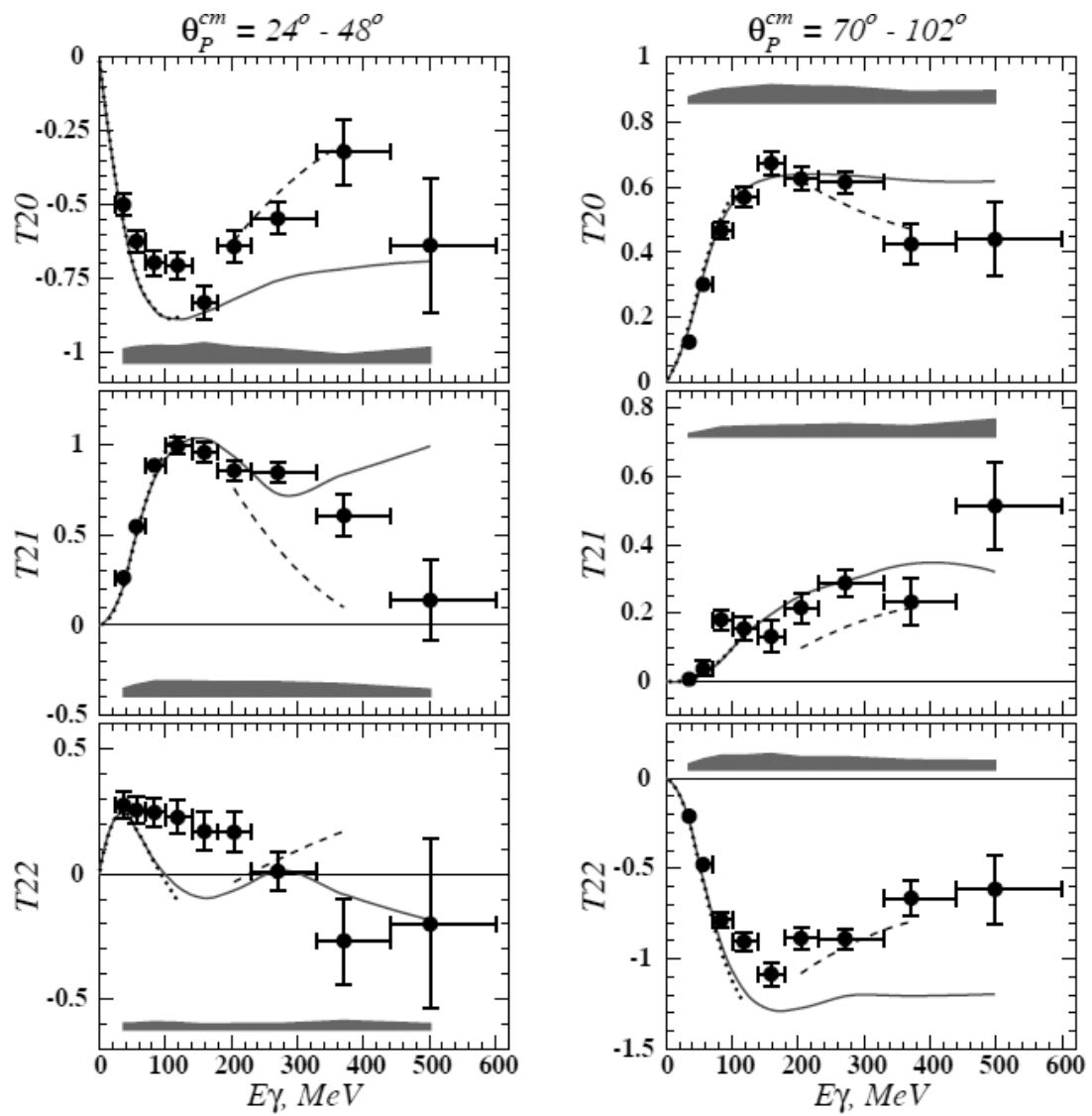

Fig. 3. Tensor analyzing powers vs. photon energy. Vertical bars are statistical uncertainties; horizontal bars indicate the bin size. Shaded bands show systematic uncertainties. Theoretical predictions are from Arenh ovel "N+MEC" (long-dashedline), "N+MEC+IC" (dash-dotted line), and "N+MEC+IC+RC" (solid line) models, from Levchuk (dotted line), and from Schwamb (shortdashed line). From Ref. 19.

$\gamma$-quantum, and $\varphi_{H}$ the angle between the polarization plane (containing the polarization axis and momentum of the photon) and the reaction plane (containing momenta of the proton and neutron). The tensor analyzing powers $T_{2 i}$ are functions of photon energy $E_{\gamma}$ and proton emission angle $\theta_{p}^{c m}$. The measurement of tensor analyzing powers $T_{20}, T_{21}$ and $T_{22}$ in deuteron PD substantially enhanced the quality and span of the existing experimental data. Only few measurements of tensor polarization observables in deuteron PD have been reported up to now. ${ }^{16-19}$ The results of these measurements enable an accurate test of available models. Theoretical calculations provide an excellent description of these polarization data below pion production threshold, while above pion production threshold a very good description of $T_{20}$ and $T_{22}$ is demonstrated by a novel approach incorporating a $\pi$-MEC retardation mechanism. 


\subsection{The HERMES experiment}

The HERMES experiment was located in the East Hall of the HERA facility at DESY. The electron/positron beams automatically became transversely polarized through a Sokolov-Ternov mechanism. ${ }^{20}$ The beam spin orientation is rotated into the longitudinal direction just upstream of HERMES, and is rotated back into the transverse direction downstream of the spectrometer. First a polarized ${ }^{3} \mathrm{He}$ internal target was used to measure the neutron spin structure function $g^{1}(n) .{ }^{21}$ Later the experiment was investigating the spin structure of the proton and neutron via deepinelastic scattering of polarized positrons/electrons from longitudinally polarized hydrogen and deuterium targets. The nucleon spin can be decomposed conceptually into the angular momentum contributions of its constituents according to the equation

$$
\left\langle s_{z}^{N}\right\rangle=\frac{1}{2}=\frac{1}{2} \Delta \Sigma+L q+J g,
$$

where the three terms give the contributions to the nucleon spin from the quark spins, the quark orbital angular momentum, and the total angular momentum of the gluons, respectively. In the measurement a value of $\Delta \Sigma=0.347 \pm 0.024 \pm 0.066$ was obtained $^{22}$ in agreement with the result of the Spin Muon Collaboration within their combined uncertainties. ${ }^{23} \mathrm{Up}$ to today it is still a mystery about what makes the spin of the nucleon. In 1998 the target was converted to one of longitudinally polarized deuterium with nuclear spin one. This allowed not only vector polarization $P_{z}$ but also tensor polarization $P_{z z}$ of the target to be produced. The latter is related to the structure function $b_{1}$ of the deuteron, which HERMES measured for the first time. This pioneer measurement has shown an anomalously large negative value of $b_{1}$ in the region $0.2<x<0.5$, although the models predict a small or vanishing value of $b_{1}$ at moderate $x .^{24}$ For the next phase, from 2001 to 2005, a transversely polarized hydrogen target was required to study transversity, the last missing leading-twist structure function of the nucleon. ${ }^{25} \mathrm{~A}$ huge statistical data collected during HERMES experiment is under analysis up to now, see e.g. Ref. 26. A dose of reports on the latest results of the analysis of the data were presented at this Symposium by A. Rostomyan, H. Marukyan and others.

\section{Experiments with Polarized Targets in the Ion Storage Rings}

\subsection{The FILTEX experiment}

The first use of an internal polarized hydrogen target in the ion ring was performed in 1992 at TSR. ${ }^{27}$ Hereafter a polarized proton target used as a spin filter (FILTer EXperiment) at TSR demonstrated a possibility to obtain a polarized circulated proton beam being initially unpolarized. ${ }^{28}$ Spin filtering using the spin-dependent part of the nucleon-nucleon interaction is the only experimentally demonstrated viable method to polarize antiprotons. This method is opening a door for new experiments at Facility for Antiproton and Ion Research (FAIR) at GSI, Darmstadt 

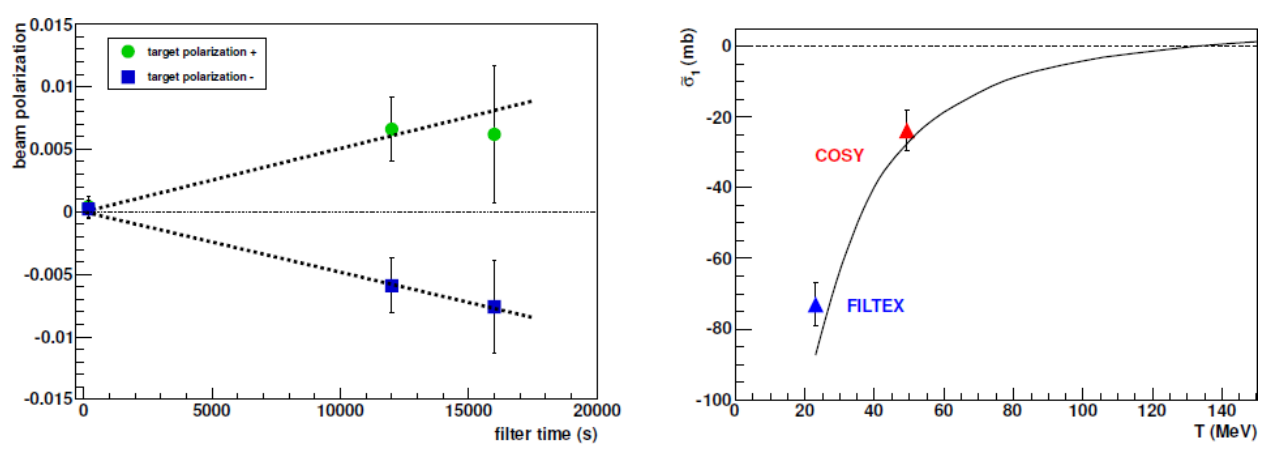

Fig. 4. Left panel: Polarization induced in the beam after filtering for different times and different signs of the target polarization. Right panel: Measured spin-dependent polarizing cross section for the interaction (only statistical errors are shown). From Ref. 29.

aimed to studying hadron structure in the interaction of polarized protons with polarized antiprotons. In 2011 the Polarized Antiproton eXperiments (PAX) Collaboration performed a successful spin-filtering test using protons at $\mathrm{Tp}=49.3 \mathrm{MeV}$ at the COSY ring, which confirms that spin filtering is a viable method to polarize a stored beam and that the present interpretation of the mechanism in terms of the proton-proton interaction is correct. ${ }^{29}$ The results of the experiment are shown in Fig. 4.

\subsection{Nuclear physics experiments}

The very unique possibilities to study nucleons interaction open when both circulated ion beam and target are polarized. An impressive program of such kind of experiments were carried out by the PINTEX group for studying proton-proton and proton-deuteron scattering and reactions between 100 and $500 \mathrm{MeV}$ at the Indiana University Cyclotron Facility (IUCF). ${ }^{30}$ An electron-cooled polarized proton or deuteron beams and polarized targets of hydrogen or deuterium were used. The experiments on $p p$ elastic scattering, pion production and to study the three-nucleon force were performed with high accuracy. The quality of the experiment ${ }^{31}$ on the elastic $p p$ scattering is illustrated in Fig. 5, right panel.

The other scientific center where similar experiments are in progress right now is a COSY - Cooler Synchrotron of the Forschungszentrum Juelich, which operates with cooled polarized and unpolarized protons and deuteron beams up to momenta of $3.7 \mathrm{GeV} / \mathrm{c}$ for the experiments with internal polarized proton and deuteron targets (EDDA, ANKE, WASA, PAX). Some results of EDDA Collaboration ${ }^{32}$ on the measurement of spin correlation parameters $A_{N N}, A_{S S}$, and $A_{S L}$ at $2.1 \mathrm{GeV}$ in $p p$ elastic scattering are shown in Fig. 6. A wide program of scientific research with polarized targets and recent results of the activity at COSY were reported at Symposium by A. Kacharava, G. Ciullo and D. Eversheim. 


\section{T. Dmitriy}
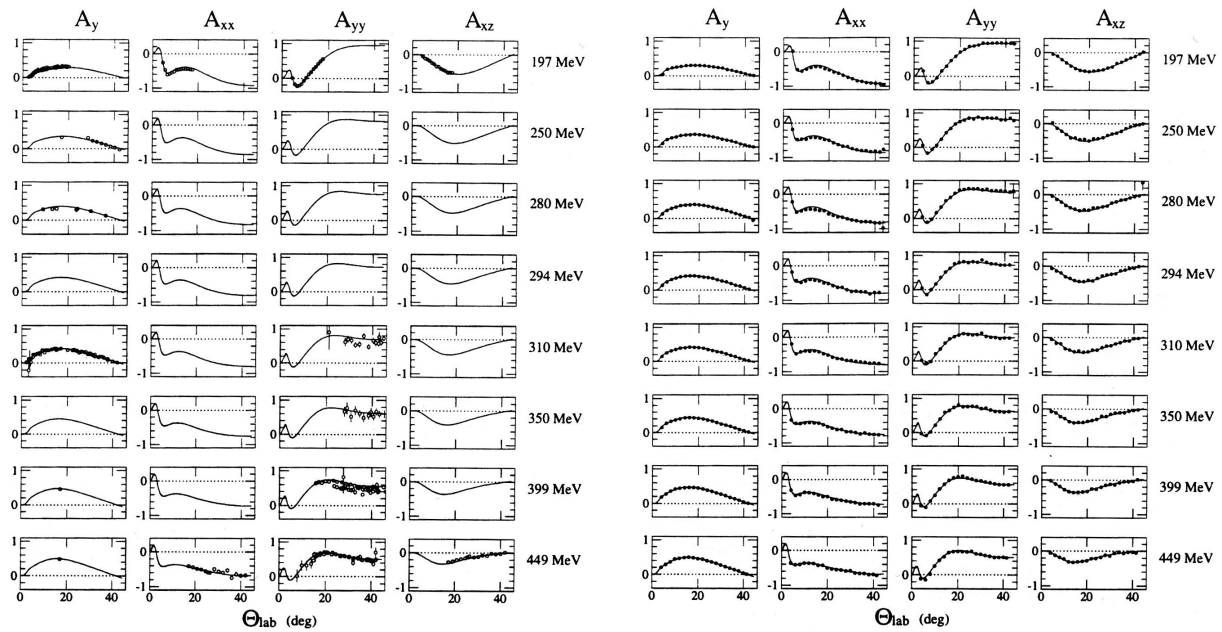

Fig. 5. Analyzing power and spin correlation coefficients as function of energy and angle. The curves are the SM97 phase shift analysis. Left panel - all previously existing data between $175 \mathrm{MeV}$ and $475 \mathrm{MeV}$ from the SAID data base. From Ref. 31.

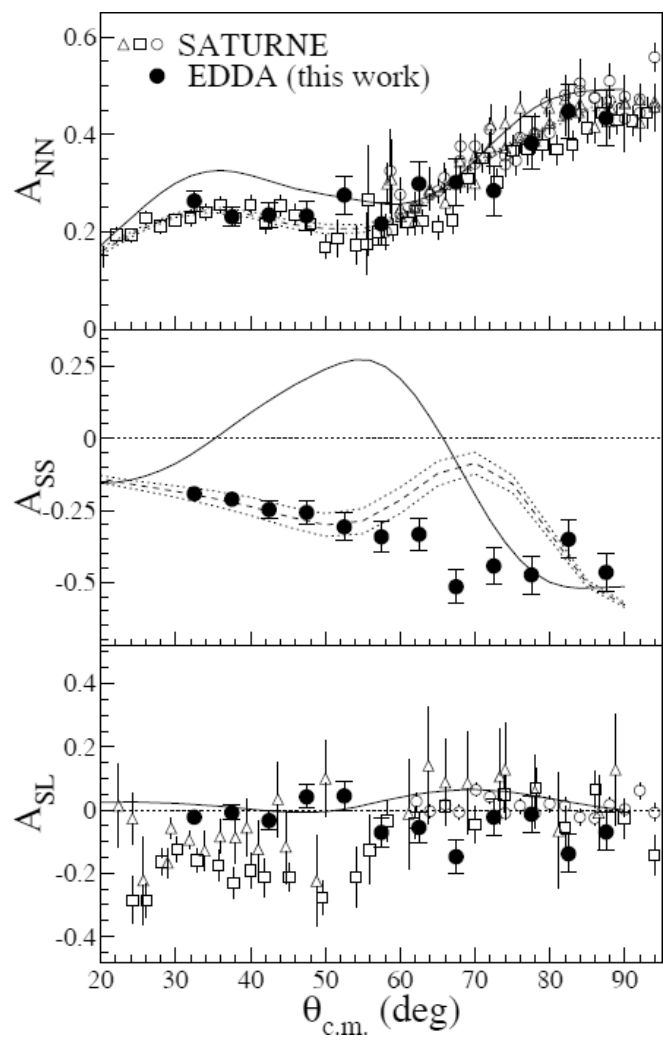

Fig. 6. Results for $A_{N N}, A_{S S}$, and $A_{S L}$ in comparison to PSA predictions. From Ref. 32. 


\section{Polarized Target for Polarimetry of the Circulated Beam}

\subsection{Polarimetry of the electron beam}

The measurement of the beam polarization in the storage rings and colliders is one of the important tasks in high-energy physics experiments. The method for measurement of an absolute extent of beam polarization in electron-positron storage rings based on MØller scattering on the internal polarized gas target was developed for the first time and successfully applied at VEPP-3 storage ring. ${ }^{33}$ A jet of deuterium atoms having atomic electrons totally polarized was used as a polarized electron target. The polarization of the electron beam was determined through the asymmetry measurement

$$
A_{N N}=\frac{N_{\uparrow \uparrow}-N_{\uparrow \downarrow}}{N_{\uparrow \uparrow}+N_{\uparrow \downarrow}}=A_{g}\left|\overrightarrow{\xi_{B}}\right|\left|\overrightarrow{\xi_{T}}\right| .
$$

Here $N_{\uparrow \uparrow}$ and $N_{\uparrow \downarrow}$ are the numbers of scattering events registered in two states of the relative polarization orientation provided by switching a sign of target polarization, the factor $A_{g}$ is less than $1 / 9$ because of a non-zero angular acceptance of the detector. $\overrightarrow{\xi_{B}}$ and $\overrightarrow{\xi_{T}}$ are the polarization vectors of the beam and the target. The measured data serve as a basis for adjusting a scenario of obtaining the polarized beams at VEPP-4M in the energy region near tau-lepton production threshold.

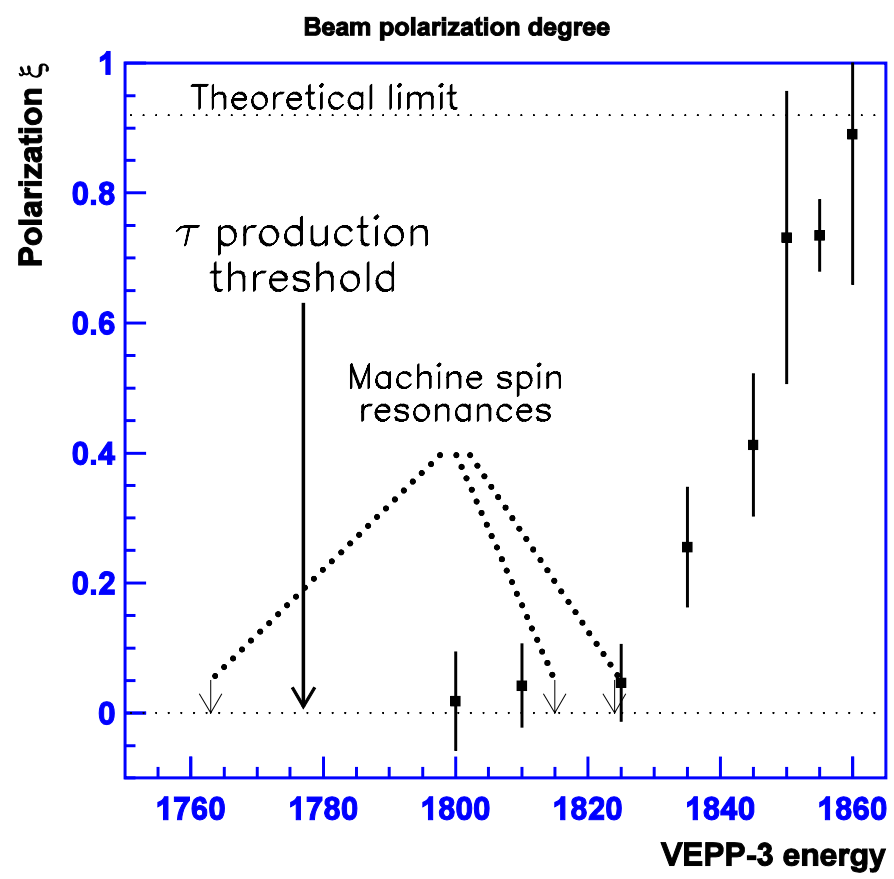

Fig. 7. Measured electron beam polarization degree vs. VEPP-3 beam energy, from Ref. 33. 


\section{T. Dmitriy}

\subsection{Polarimetry of the proton beam}

The other application of the polarized atomic hydrogen gas jet is for absolute polarization measurement of the circulated protons beams at RHIC. ${ }^{34}$ The measurement is based on the elastic proton-proton scattering in the Coulomb-Nuclear Interference (CNI) region. $A_{N}$ is a measure of the left-right asymmetry of the cross section in the scattering plane normal to the beam or target polarization. The $p p$ elastic scattering process is 2-body exclusive scattering with identical particles. $A_{N}$ for the target polarization and the beam polarization should be the same as shown in Equation (6)

$$
A_{N}=-\frac{\varepsilon_{\text {target }}}{P_{\text {target }}}=\frac{\varepsilon_{\text {beam }}}{P_{\text {beam }}}
$$

$\varepsilon_{\text {target }}$ is raw asymmetry for the $p p$ elastic scattering for the transversely polarized proton target and $P_{\text {target }}$ is a polarization of the target. The first precise measurement of the analyzing power $A_{N}$ in $p p$ elastic scattering in the CNI region was performed at RHIC with polarized hydrogen jet the proton polarization of which was well known. ${ }^{35}$ The analyzing power $A_{N}$ in $p p$ elastic scattering in the CNI region was measured to be big enough and weakly depend on the energy for a wide range from $24 \mathrm{GeV}$ to $250 \mathrm{GeV}$. The beam polarization can be found from the equation

$$
P_{\text {beam }}=-P_{\text {target }} \frac{\varepsilon_{\text {beam }}}{\varepsilon_{\text {target }}} .
$$

The average beams polarization in RHIC in RUN11 measured by the polarized jet target $^{36}$ is shown in Fig. 8. The proton polarimetry current status and future plans were reported at Symposium by Y. Makdisi.

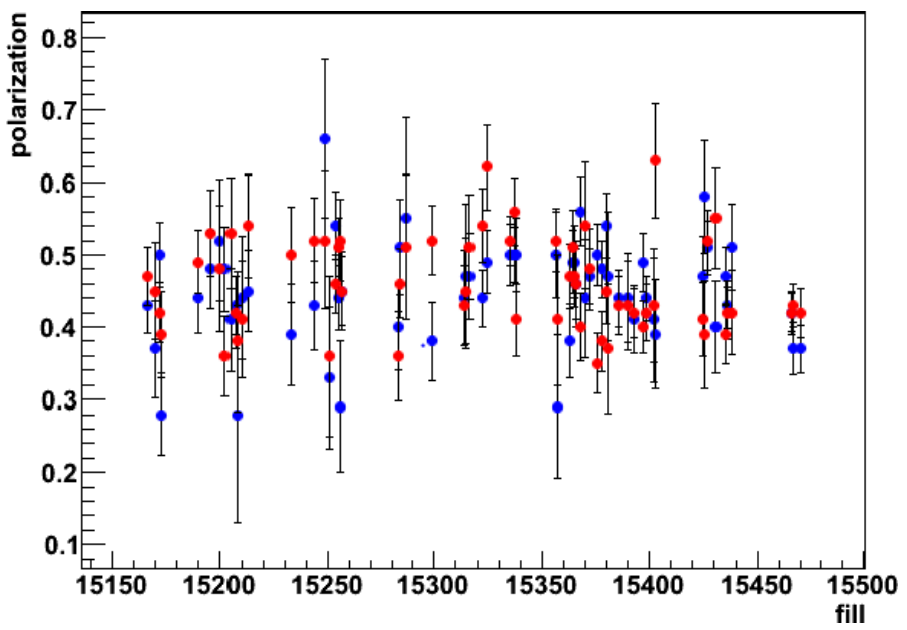

Fig. 8. H-jet polarization measurements, Run 2011. From Ref. 36. 


\section{Electromagnetic Form Factors of the Proton}

Elastic electron-nucleon scattering is one of the experimental tool for accessing the information on the internal structure of the nucleon. Two structure functions, form factors, $G_{E}\left(Q^{2}\right)$ and $G_{M}\left(Q^{2}\right)$ describe the internal structure of the proton. For a long time, the only experimental method to measure form factors was the Rosenbluth method or longitudinal-transverse separation, based on the well-known description of the elastic ep scattering in the one-photon exchange approximation. Another method of direct measuring the ratio $G_{E} / G_{M}$, the so-called polarization transfer method, was proposed in the mid of last century, ${ }^{37}$ but could be realized only several decades ago. Surprisingly a clear discrepancy was observed between the results obtained by these two methods for the range of $Q^{2} \geq 1 \mathrm{GeV}^{2}$, see Fig. 9. It was suggested that the one possible explanation of such a discrepancy is the omission of the two-photon exchange (TPE) contribution in the Rosenbluth formulae. Inclusion of the two-photon exchange correction in the Rosenbluth process may noticeably reconcile the results of two methods. ${ }^{38}$ There are three new experiments aimed a precise measurement of the TPE contribution to the elastic $e p$ scattering. Two of them (at VEPP-3 storage ring in Novosibirsk, Russia ${ }^{39}$ and the OLYMPUS at DESY, in Hamburg, Germany ${ }^{40}$ ) used internal hydrogen target and electron/positron beams circulated in the rings. The third experiment has been performed in the Jefferson Lab, USA with liquid hydrogen target and detecting the scattered particles by CLAS detector. ${ }^{41}$ The experimentally measured quantity is the ratio $R=\sigma\left(e^{+} p\right) / \sigma\left(e^{-} p\right)$ of the elastic $\sigma\left(e^{+} p\right)$ and $\sigma\left(e^{-} p\right)$ scattering cross sections. The desired hard TPE contribution to the reaction $\delta_{2 \gamma}$ was determined from $R$ after taking into account the first order radiative corrections. The results presented as the $R_{2 \gamma}=\left(1-\delta_{2 \gamma}\right) /\left(1+\delta_{2 \gamma}\right)$ and some theoretical predictions for $R_{2 \gamma}$ are shown in Fig. 10. The results obtained show evidence of a significant hard TPE

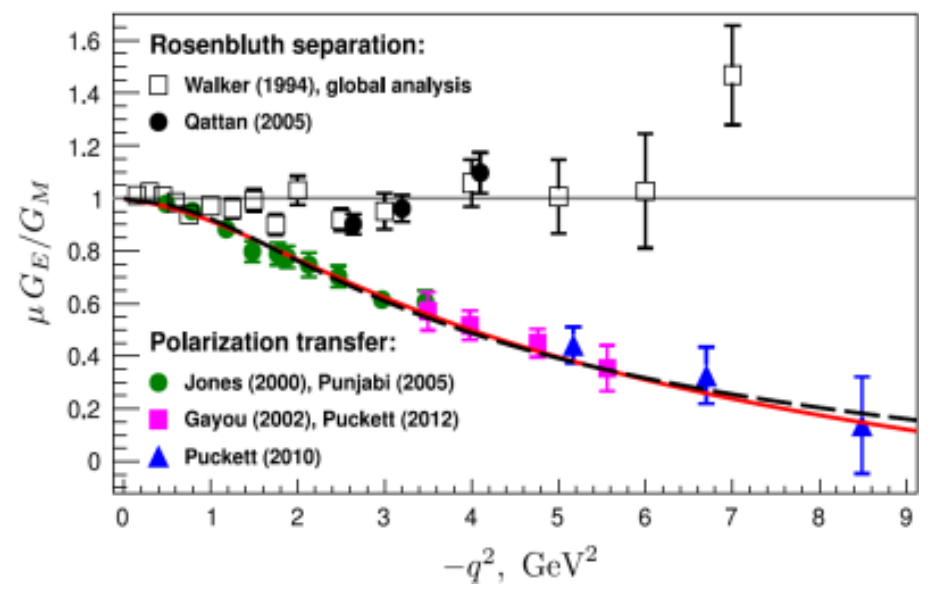

Fig. 9. The ratio $\mu G_{E} / G_{M}$ obtained using different methods. 

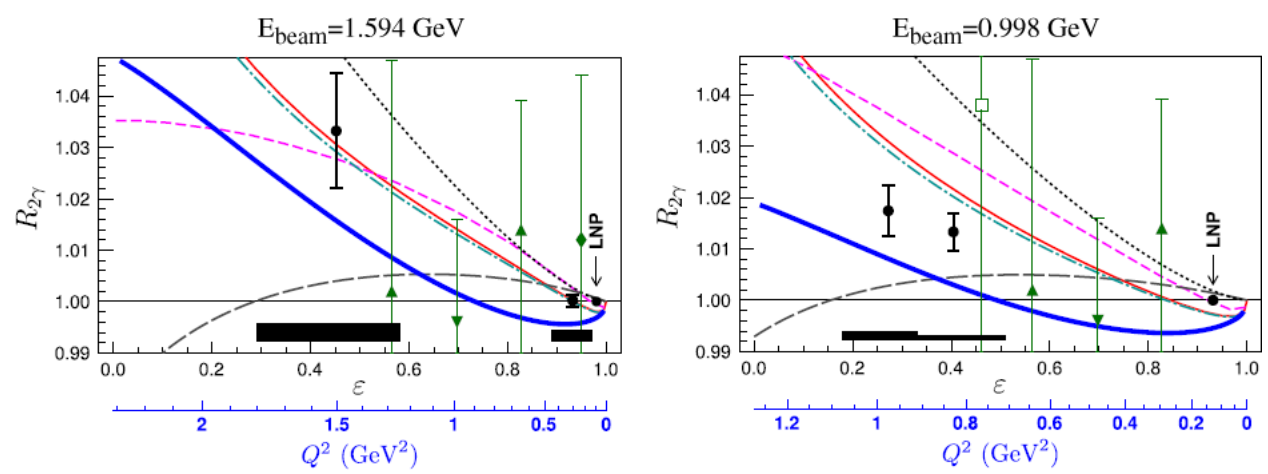

Fig. 10. The data of Novosibirsk experiment (closed circles), earlier measurements and some theoretical predictions (curves) for the ratio $R_{2 \gamma}$ as a function of $\varepsilon$ or $Q^{2}$. From Ref. 39 .

effect. They are in moderate agreement with several theoretical predictions of TPE contribution explaining the form factor discrepancy at high $Q^{2}$ values.

The situation with the electromagnetic form factors of the proton was discussed at this Symposium in the reports of B. Briscoe, G. Cates and I. Cloet.

\section{Conclusion}

During the two last decades numerous experiments based on polarized gas targets have been performed in the storage rings of charged particles. Polarized hydrogen, deuterium and ${ }^{3} \mathrm{He}$ gas targets have opened of new possibilities in physical experiments and enable to get data unachievable earlier. A related activity in some laboratories is directed on the preparation of new experiments and creation of more dense polarized targets needed for future experiments. It seems that the ABS technique employing the storage cell for polarized atoms has reached the limit. New ideas being discussed involve the compression of polarized molecules obtaining by nuclear Stern-Gerlach method or made by recombining of polarized atoms. ${ }^{42,43}$

\section{References}

1. G. I. Budker et al., Yadernaya fisika 6, 775 (1967).

2. V. F. Dmitriev et al., Nuclear Physics A464, 237 (1987).

3. R. Gilman and F. Gross, J. Phys. G 28, R37 (2002).

4. M. Garcon and J. W. Van Orden, Adv. Nucl. Phys. 26, 293 (2001).

5. V. F. Dmitriev et al., Phys. Lett. 157B, 143 (1985).

6. R. Gilman et al., Phys. Rev. Lett. 65, 1733 (1990).

7. D. M. Nikolenko et al., Phys. Rev. Lett. 90, 072501 (2003).

8. Ferro-Luzzi et al., Phys. Rev. Lett. 77, 2630 (1996).

9. M. Bouwhuis et al., Phys. Rev. Lett. 82, 3755 (1999).

10. C. Zhang et al., Phys. Rev. Lett. 107, 252501 (2011).

11. D. Abbott et al., Eur. Phys. J. A 7, 421 (2000).

12. I. Passchier et al., Phys. Rev. Lett. 82, 4988 (1999).

13. E. Geis et al., Phys. Rev. Lett. 101, 042501 (2008). 
14. C. B. Crawford et al., Phys. Rev. Lett. 98, 052301 (2007).

15. R. Gilman and F. Gross, J. Phys. G: Nucl. Part. Phys. 28, 37 (2002).

16. M. V. Mostovoy et al., Phys. Lett. B188, 181 (1987).

17. K. H. Althoff et al., Z Phys. C43, 375 (1989).

18. S. I. Mishnev et al., Phys. Lett. B302, 23 (1993).

19. I. A. Rachek et al., Phys. Rev. Lett. 98, 182303 (2007).

20. A. A. Sokolov and I. M. Ternov, Sov. Phys. Doklady 8, 1203 (1964).

21. K. Ackerstaff et al., Phys. Lett. B404, 383 (1997).

22. A. Airapetian et al., Phys. Rev., D71, 012003 (2005).

23. SMC Collaboration, B. Adevaet et al., Phys. Lett. B420, 180 (1998).

24. A. Airapetian et al., Phys. Rev. Lett. 95, 242001 (2005).

25. A. Airapetian et al., Phys. Rev. Lett. 94, 012002 (2005).

26. http://www-hermes.desy.de/notes/pub/publications.html

27. M. Duren et al., Nucl. Instrum. Methods A 322, 13 (1992).

28. F. Rathmann et al., Phys. Rev. Lett. 71, 1379 (1993).

29. C. Weidemann for the PAX collaboration. EPJ Web of Conferences 66, 11039 (2014).

30. http://ceem.indiana.edu/documents/Pintex Home Page @ IUCF.pdf

31. B. V. Przewoski et al., Phys. Rev. C 58, 1897 (1998).

32. F. Bauer et al., Phys. Rev. Lett. 90, 142301 (2003).

33. M. V. Dyug et al., Nucl. Instrum. Methods A 536, 338 (2005).

34. A. Zelenski et al., Nucl. Instrum. Method. A 536, 248 (2005).

35. H. Okada et al., Physics Letters B 638, 450 (2006).

36. A. Zelenski, Polarimetry at RHIC, in Conf. Proc. PSTP2011, eds. K. Grigoriev, P. Kravtsov and A. Vasilyev (Russia, St. Petersburg, 2011), p. 39.

37. A. I. Akhiezer and M. P. Rekalo, Sov. Phys. Dokl. 13, 572 (1968).

38. C. E. Carlson and M. Vanderhaeghen, Annu. Rev. Nucl. Part. Sci. 57, 171 (2007).

39. I. A. Rachek et al., arXiv:1411.7372.

40. R. Milner et al., (OLYMPUS Collaboration), Nucl. Instrum. Methods A 741, 1 (2014).

41. D. Adikaram et al., (CLAS Collaboration), arXiv:1411.6908.

42. D. Toporkov, Proceedings of Sciences (PSTP 2013) 064.

43. R. Engels et al., Rev. Sci. Instrum. 85, 103505 (2014). 